Sociologie et sociétés

\title{
Quand les bonnes intentions se substituent à l'analyse critique
}

Réplique à Louise Nadeau

\section{When Good Intentions Take the Place of Critical Analysis}

Reply to Louise Nadeau.

\section{David COHEN}

Volume 28, numéro 2, automne 1996

Technologies médicales et changement de valeurs

URI : https://id.erudit.org/iderudit/001139ar

DOI : https://doi.org/10.7202/001139ar

Aller au sommaire du numéro

Éditeur(s)

Les Presses de l'Université de Montréal

ISSN

0038-030X (imprimé)

1492-1375 (numérique)

Découvrir la revue

Citer cet article

COHEN, D. (1996). Quand les bonnes intentions se substituent à l'analyse critique : réplique à Louise Nadeau. Sociologie et sociétés, 28(2), 39-43. https://doi.org/10.7202/001139ar 


\section{Quand les bonnes intentions se substituent à l'analyse critique.} Réplique à Louise Nadeau

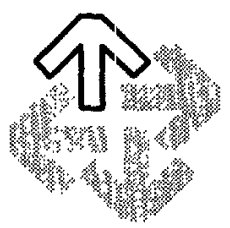

\section{DAVID COHEN}

Je remercie Louise Nadeau d'avoir pris le temps de rédiger sa note critique de mon texte sur les «nouveaux » médicaments de l'esprit, mais je regrette qu'elle étale ainsi sa compréhension erronée du texte, une grave méconnaissance du domaine psychiatrique ainsi qu'un réalisme naïf étonnant.

MANICHÉISME DE L'ARTICLE OU DÉSÉQUILIBRE DE POUVOIR DANS LE SYSTÈME?

Louise Nadeau retient l'idée générale d'une « conspiration entre les trusts pharmaceutiques et les médecins psychiatres [...] alliés contre ceux qui les consultent pour de la détresse psychologique ", idée qui exprime un manichéisme sous-jacent, un dualisme du «Bien et [du] Mal, [de] la Victime et [du] Bourreau ».

Je suis au contraire convaincu que mon texte fait ressortir la complexité du système de santé mentale et la variété des intérêts du grand nombre d'acteurs impliqués (patients ou clients, psychiatres ou médecins généralistes, familles, établissements de santé, tiers-payants, compagnies pharmaceutiques, chercheurs, médias, l'État, etc.). Ceci dit, face aux «personnes qui consultent », le pouvoir des médecins et des laboratoires pharmaceutiques est, incontestablement, démesuré et, du point de vue du fonctionnement démocratique de la société, injustifié (McCubbin et Cohen, 1996). Ainsi, quand Louise Nadeau estime que l'utilisation de neuroleptiques toxiques est due à la difficulté des patients de former un lobby et d'influencer la recherche, elle soutient précisément l'idée que les patients les plus démunis ne voient pas leurs intérêts protégés dans le système actuel.

Cohen et McCubbin (1990) ont montré en détail comment les acteurs impliqués dans le processus décisionnel qui aboutit à la prescription de neuroleptiques répondent à divers incitatifs et contraintes. Le système est ainsi fait qu'il pousse à choisir les neuroleptiques, à sous-estimer leurs risques et à exagérer leurs bienfaits. Si on ajoute à cela un rapport de forces déséquilibré, le système perd en efficacité, la plus grande partie des coûts étant alors assumée par des acteurs qui n'ont ni le désir ni le pouvoir de les imposer. 


\section{CONSPIRATION OU «PSEUDOSCIENCE NORMALE »?}

Ainsi, l'idée d'une «conspiration » est simpliste. Les faits que je décris dans mon texte découlent surtout d'une série d'erreurs cognitives en psychiatrie biologique renforcées par le milieu ambiant, selon un processus que Kuhn (1970) a décrit en détail. Par exemple, on ne décrirait pas la collusion de l'Église et des astronomes à l'époque de Galilée soutenant que la Terre était au centre de l'univers comme une conspiration ; ils exprimaient là leur appui à un paradigme scientifique générant des travaux de «science normale » et leur résistance naturelle au changement.

Dans une discussion scientifique, une fois les termes du paradigme balisés, pourquoi ne pas utiliser les résultats des recherches s'inscrivant dans le paradigme ? D'ailleurs, de quels autres outils disposons-nous? Les recherches astronomiques avant Copernic étaient utiles, puisqu'elles ont révélé, entre autres, les anomalies qui ont éventuellement renversé le paradigme sur lesquelles elles reposaient. Mutatis mutandis, les recherches générées en psychiatrie biologique sont très utiles - mais à quoi ? À mon avis, et celui d'un nombre grandissant de chercheurs (voir Ross et Pam, 1995), elles font ressortir les biais idéologiques du bioréductionnisme, l'absence de corrélation entre les observations biologiques et les diagnostics psychiatriques, l'incapacité de prédire ces observations biologiques, le pouvoir remarquable du placebo, la tendance à publier des résultats statistiquement significatifs mais sans aucune portée clinique, le refus ou l'incapacité de considérer le traumatisme infantile grave comme un facteur étiologique majeur, etc., etc.

Je ne vois donc pas de «bons » et de «méchants » chercheurs (ou prescripteurs ou vendeurs de médicaments), comme Louise Nadeau m'en accuse dans sa note ; je vois seulcment des travaux de recherche (ou activités thérapeutiques ou commerciales) qu'il faut scruter avec un esprit critique et une position intellectuelle explicite.

\section{MA POSITION}

Quelle est donc ma position et pourquoi donc ma «charge contre la psychiatrie»? En premier lieu, je suis d'avis que la majeure partie de la psychopathologie dans notre culture représente la réponse normale de l'individu biologiquement et génétiquement normal au traumatisme chronique dans l'enfance et à son renforcement plus tard par un manque de pouvoir plus ou moins évident. Les questionnaires structurés actuellement disponibles et les méthodes épidémiologiques classiques nous permettent de tester cette hypothèse (Mirowsky et Ross, 1989). Si cet avis est justifié, la psychiatrie biologique dans sa forme actuelle constitue un gaspillage de ressources majeur; celles-ci devraient être redirigées vers la psychobiologie normale du traumatisme et de l'impuissance. Elles produiraient rapidement des résultats significatifs sur les plans biologique et clinique, à l'intérieur d'un paradigme biopsychosocial.

En second lieu, je laisse à Colin Ross, psychiatre, consultant pour l'industrie pharmaceutique et auteur de recherches en psychiatrie biologique, le soin d'exprimer ma charge contre cette discipline : « La psychiatrie biologique n'a pas fait une seule découverte pertinente sur le plan clinique ces dernières dix années, en dépit de centaines de millions de dollars investies sur la recherche. [...] [L]e fardeau de la preuve doit revenir à la psychiatrie biologique, de décrire un seul résultat découlant du travail bioscientifique de psychiatres ayant une quelconque application ou pertinence clinique directe " (Ross et Pam, 1995, p. 116; c'est nous qui traduisons).

Je suis donc attristé de voir Louise Nadeau porter atteinte à la qualité de sa critique quand elle évoque les souffrances et la dangerosité présentées par des personnes portant divers diagnostics, ou les intentions nobles des soignants, comme la preuve que le problème fondamental du manque de validité diagnostique en psychiatrie et la réduction du discours sur le trouble mental aux seuls symptômes accessibles aux médicaments se résument à « une erreur dans la classification des troubles mentaux »! Les gens souffrent et dérangent, c'est un fait. 
Que la psychiatrie ait toujours rempli le double mandat de soins et de contrôle en est un autre. Qu'il faille développer un système diagnostique fiable et opérationnel, c'est une tout autre question. Que ce système doive être de type DSM en est encore une autre! Amalgamer toutes ces questions en une seule - sous prétexte que de pauvres gens souffrent et que de nobles thérapeutes veulent les aider - illustre le type d'erreurs de raisonnement prévalent dans le discours psychiatrique actuel et, je le déplore, dans la critique de Louise Nadeau. La valeur de cette stratégie cognitive est cruciale, puisqu'elle permet de maintenir le système dans son homéostase dysfonctionnelle.

Quant à m'imputer l'idée que la schizophrénie serait une "vue de l'esprit ", et non pas une « entité diagnostique », Louise Nadeau est encore hors propos. Le seul critère possible pour décider si un individu souffre de schizophrénie est clinique; la fiabilité du concept de schizophrénie est donc limitée par la valeur de la statistique (kappa entre 0,6 et 0,7; Kirk et Kutchins, 1992) exprimant l'accord entre deux juges essayant de l'appliquer. Même si la plupart des cliniciens estiment qu'un facteur biologique est impliqué dans la schizophrénie, il n'est pas clair qu'un tel facteur, s'il existe, sera la cause ou l'effet. Les modèles restent spéculatifs. Selon l'état actuel des connaissances, il est concevable qu'un facteur environnemental assez nocif puisse mener à une décompensation psychotique sans aucune prédisposition physique. Rien ne nous permet encore de justifier des affirmations dogmatiques, telles qu'on en retrouve partout, quant à la transmission génétique, la prédiposition constitutionnelle ou le mode d'action biologique (Ross et Pam, 1995).

Quant à l'efficacité des thérapies psychosociales dans les troubles mentaux, je renvoie Louise: Nadeau aux méta-analyses de Karon (1989), aux études avec groupe témoin de Mosher et ses collègues (voir Mosher et Burti, 1989) ainsi qu'aux résultats empiriques plus récents de Ciompi et coll. (1992) pour affirmer et insister que, dans l'état actuel des connaissances, le traiternent psychosocial de la psychose dans de petites structures résidentielles, sans médicaments neuroleptiques ou avec des doses minimes rarement utilisées, reste équivalent ou supérieur à l'hospitalisalisation et la médication. De plus, j'accepte sans réserve la conclusion de Karon (1989) : « Malheureusement, ce qui semble couramment imposer le type de traitement à administrer, ce n'est pas des visées scientifiques, mais plutôt des facteurs politiques et économiques, et un grand souci de rentabilité à court terme. » (Karon 1989, p. 146 ; c'est nous qui traduisons.)

\section{LE MANQUE DE DONNÉES : ON POURRAIT QUASIMENT PARLER DE CONSPIRATION ...}

Ayant fourni, dans la version finale de mon manuscrit, diverses sources pour appuyer mes affirmations sur l'augmentation de la consommation de psychotropes et, en particulier, celle du Prozac; sur la substitution aux anxiolytiques de nouveaux antidépresseurs ; sur les côuts largement supérieurs des nouveaux médicaments, je m'étonne que Louise Nadeau ne sache pas que les données sur le marché des psychotropes sont encore mieux gardées que les secrets de la défense nationale. À moins d'avoir un accès direct à des sources de données commerciales comme celles d'IMS, il est extrêmement difficile, sinon impossible, de connîitre ces chiffres. Même les pouvoirs publics n'y ont qu'un accès limité et doivent se contenter des données colligées à partir des renseignements dans les caisses d'assurance-santé (qui ne touchent, au Canada, que certaines tranches de la population). À titre d'exemple récent, suite à des démarches futiles auprès du manufacturier et du ministère de la Santé fédéral, ce n'est qu'auprès de la Régie d'assurance-maladie du Québec que j'ai pu obtenir des chiffres sur la consommation de méthylphénidate (Ritalin) - mais seulement chez les enfants dont les familles se voient remboursés les médicaments.

Cela dit, si Louise Nadeau n'estime pas que le Prozac est devenu - comme produit commercial et référent culturel - presque aussi banal que le Kleenex, je laisse aux lecteurs le soin d'évaluer ses connaissances du domaine pharmaceutique. 


\section{L'INFLUENCE DE L'INDUSTRIE PHARMACEUTIQUE : EST-IL POSSIBLE DE NE PAS LA VOIR?}

En soi, la question des profits de l'industrie pharmaceutique a peu d'intérêt, surtout si celle-ci améliore suhstantiellement le bien-être des citoyens. Il est plus pertinent de poser des questions sur la nature de cette industrie (essentiellement oligopolistique), sur les externalités qu'elle génère et qui en assume les coûts, et sur l'influence qu'elle exerce sur les politiques publiques et les professionnels de la santé. Sur ce dernier sujet, Louise Nadeau m'accuse de tenir des « propos pamphlétaires ».

Nous avons de la chance de posséder les résultats d'une enquête unique, menée en France par Édouard Zarifian, professeur de psychiatrie, à la demande du ministre de la Santé et tout récemment rendue publique (un long article principal sur ce rapport apparait dans le magazine L'Express du 28 mars 1996). Selon la dépêche de l'Agence de presse médicale du 29 mars 1996 par Hélène Mauduit, où de longs extraits du rapport sont cités, Zarifian dénonce un discours universitaire psychiatrique « à la remorque de l'industrie pharmaceutique ». « La prescription des médicaments psychotropes évolue dans un environnement où les discours académiques sont en parfaite concordance avec les discours promotionnels de l'industrie pharmaceutique et ne proposent rien d'autres que la prescription. » Zarifian déplore le biais des études réalisées ou diffusées en France. Son constat s'appuie sur l'omniprésence de l'industrie pharmaceutique : «Pratiquement toutes les études cliniques, épidémiologiques, médico-économiques, sont suscitées, financées et exploitées au plan statistique de manière autonome par l'industrie ou par des sous-traitants qu'elle rémunère. » Il résulterait de cette dépendance une absence de débat sur plusieurs sujets gênants pour l'industrie, dont les liens entre experts et laboratoires. Zarifian va même jusqu'à dénoncer des manipulations qui « visent à gonfler la fréquence des pathologies pour justifier des volumes importants de prescriptions ». La situation dont Zarifian dresse un constat si accablant dans son rapport de 300 pages est celle de la France. J'invite l'industrie pharmaceutique à prouver que la situation est différente ailleurs. Et jusqu'à preuve du contraire, je suggère que les faits nous forcent à accepter les conclusions du professeur Zarifian (qu'elles apparaissent ou non «pamphlétaires » aux yeux de Louise Nadeau).

\section{LA SCIENCE NEUTRE?}

Louise Nadeau avoue sa difficulté à ne pouvoir « faire la distinction entre les propos du scientifique ... [et ceux du] pamphlétaire qui dénonce $»$. C'est sans doute le commentaire qui m'a le plus surpris. Pourquoi donc faut-il faire cette distinction ? N'est-il pas plus important de juger la qualité d'une argumentation sur sa logique, les preuves apportées, etc. ? Un scientifique qui « dénonce » ou ironise ne serait-il plus un scientifique ? Questions qui méritent certainement d'être débattues - mais pour moi, ce propos renvoie à un modèle illusoire (sinon dangereux) de la science ou du scientifique «neutre». J'ai présenté de nombreuses données indiscutables, que j'ai évaluées à la lumière des mes connaissances et de mon sens critique. Je me suis efforcé de rendre ma position de base transparente. Ma première obligation scientifique est d'être honnête et rigoureux ; ma seconde est de dire les choses comme je les vois.

En terminant, je me permets de réitérer une thèse centrale de mon article, qu'aucun des propos de Louise Nadeau n'a, à mon avis, affaiblie : la perception erronée de l'efficacité des médicaments psychotropes de cette fin de siècle est pour beaucoup le résultat de l'effet biologique formidable (mais non étudié !) du placebo et d'une brillante stratégie de marketing soutenue par les adhérents de la psychiatrie biologique, qui voient dans le médicament une source inestimable d'appui - d'autant que cette discipline ne peut rendre compte du trouble mental et reste limitée sur le plan intellectuel et clinique. 


\section{BIBLIOGRAPHIE}

CIOMPI, Luc, DAUWALDER, Hans-Peter, MAIER, Christian, AEBI, Elisabeth, TRÜTSCH, Karl, KUPPER, Zeno et RUTISHAUER, Charlotte (1992), "The Pilot Project "Soteria Berne" : Clinical Experiences and Results », Bititish Journal of Psychiatry, vol. 161 (supplément 18), pp. 145-153.

COHEN David et McCUBBIN, Michael (1990), « The Political Economy of Tardive Dyskinesia : Asymmetries in Power and Responsibility », Journal of Mind and Behavior, vol. 11, pp. 455-474.

KARON, Bertram (1989), « Psychotherapy Versus Medication for Schizophrenia : Empirical Comparisons », in S. Fisher, R. Greenberg (dir.), The Limits of Biological Treatments for Psychological Distress : Comparisons with Psychotherapy and Placebo, Hillsdale, New Jersey, Lawrence Erlbaum Associates, pp. 105-150.

KIRK, Stuart et KUTCHINS, Herb (1992), The Selling of DSM, Chicago, Aldine de Gruyter.

KUHN, Thomas (1970), The Structure of Scientific Revolutions ( $2^{\mathrm{e}}$ édition révisée), Chicago, University of Chicago Press. MCCUBBIN Michael et COHEN, David (1996), « Extremely Unbalanced : Interest Divergence and Power Disparities between Clients and Psychiatry *, International Joumal of Law and Psychiatry, vol. 19, n 1, pp. 1-25.

MIROWSKY, John et ROSS, Catherine (1989), Social Causes of Psychological Distress, Chicago, Aldine de Gruyter. MOSHER, Loren et BURTI, Lorenzo (1989), Community Mental Health : Principles and Practice, New York, Basic. ROSS, Colin et PAM, Alvin (1995), Pseudoscience in Biological Psychiatry : Blaming the Body, New York, Wilcy. 